\title{
Similarity grouping and repetition blindness are both influenced by attention
}

\author{
Bianca de Haan ${ }^{1 *}$ and Chris Rorden ${ }^{2}$ \\ 1 Section Neuropsychology, Center of Neurology, Hertie-Institute for Clinical Brain Research, University of Tuebingen, Tuebingen, Germany \\ Department of Communication Sciences and Disorders, University of South Carolina, Columbia, DC, USA
}

Edited by:

Leon Y. Deouell, The Hebrew University of Jerusalem, Israel

Reviewed by:

Dominique Lamy, Tel Aviv University,

Israel

Bettina Olk, Jacobs University,

Germany

\section{*Correspondence:}

Bianca de Haan, Section

Neuropsychology, Center of Neurology,

Hertie-Institute for Clinical Brain

Research, University of Tuebingen

Hoppe-Seyler-Strasse 3, D-72076

Tübingen, Germany.

e-mail: bianca.de-haan@klinikum.

uni-tuebingen.de
Previous studies have reported seemingly conflicting results regarding how the amount of stimulus similarity between two simultaneously presented target stimuli impacts perceptual performance. There are many reports of 'repetition blindness', where individuals do worse when shown two similar stimuli relative to two different stimuli. On the other hand, there are reports of 'similarity grouping', where participants perform better when identifying two similar objects relative to two different objects. This manuscript posits that repetition blindness and similarity grouping coexist and can be elicited in the same subjects in a single task. This not only explains the previous opposite effects of stimulus similarity on task performance, but also provides a unique opportunity to directly compare these opposite effects of stimulus similarity with respect to susceptibility to a modulating factor. Since previous studies have provided inconclusive results on whether attentional relevance can modulate the effect of stimulus similarity on task performance, the current manuscript aims to compare repetition blindness and similarity grouping with respect to their susceptibility to attentional relevance. The results of the first experiment confirmed that both repetition blindness and similarity grouping can be elicited in the same experiment, suggesting that repetition blindness and similarity grouping coexist. The results of the second experiment suggest that both repetition blindness and similarity grouping can be modulated by attentional relevance. These results support the explanation of repetition blindness as a token individuation failure. Furthermore, these results suggest that supposedly pre-attentional grouping mechanisms might not operate as independently from top-down attentional modulations as traditionally thought.

Keywords: attention, repetition blindness, perceptual grouping, pre-attentive processing, task relevance, stimulus similarity

\section{INTRODUCTION}

The human visual system is often bombarded with a potentially overwhelming number of stimuli. Extensive research has revealed that the brain employs a mechanism to selectively process relevant visual events and ignore irrelevant visual events. One theory that aims to explain how the brain achieves this visual selection is the theory of biased competitive interactions (Desimone and Duncan, 1995; Duncan et al., 1997; Desimone, 1998; Duncan, 1998). According to this theory, the multiple neural representations in the brain associated with the stimuli in the visual field compete for limited processing resources. Furthermore, this competition between multiple stimulus representations can be biased both by bottom-up and top-down influences. One factor that can bias these competitive interactions with striking consequences is unilateral brain damage which often results in a neuropsychological deficit known as extinction (Becker and Karnath, 2007). Patients suffering from visual extinction can detect a single target stimulus at any spatial location. However, when two target stimuli are presented simultaneously, patients are impaired at perceiving the contralesional item. In other words, the contralesional target stimulus is extinguished when an ipsilesional target stimulus is present. According to the theory of biased competitive interactions, the competition for limited processing resources is biased in extinction patients by the unilateral brain damage which results in a weaker neural representation for contralesional target stimuli. The consequence is that in competitive situations, the weaker neural representation of the contralesional target stimulus is at a disadvantage when compared to the stronger neural representation of the ipsilesional target stimulus and will usually lose the bid for attention.

Previous studies performed with both neurologically healthy subjects and neurological patients suffering from extinction suggest that depending on the circumstances, stimulus similarity between two simultaneously presented target stimuli can either improve or hinder perception. Several studies have found improved performance when two target stimuli are perceptually identical as compared to when two target stimuli are perceptually different (Vuilleumier and Rafal, 2000, experiment 1; Ward et al., 1994). Multiple identical stimuli tend to be perceived as a single perceptual group in line with the principle of similarity grouping (Wertheimer, 1923; Koffka, 1999). In accordance with the proposal from Desimone and Duncan (1995) that competitive interactions should occur mostly between perceptual groups and not within perceptual groups, similarity grouping increases the likelihood that a stimulus representation gains access to processing resources by making the multiple stimulus representations non-competing allies in the bid for visual selection. Similarity 
grouping has been associated with neural activity in the occipital and temporal cortices (Han et al., 2002, 2005a,b) in agreement with the idea that grouping processes operate at an early stage in the visual processing stream to organise to visual input for subsequent (attentional) processing. Interestingly, the influence of stimulus similarity between multiple targets, as opposed to similarity between targets and nontargets or similarity between multiple nontargets, on perceptual performance has typically been studied in neurological patients suffering from visual extinction. These extinction patients show improved performance expressed by reduced extinction severity when presented with two identical targets as compared to performance when presented with two different targets. Whether and to what extent this observation can be generalized to neurologically healthy subjects remains untested.

On the other hand, numerous studies in both extinction patients and neurologically healthy subjects have reported that perceptual performance declines when two target stimuli are perceptually identical as compared to when they are perceptually different (Baylis et al., 1993; Kanwisher et al., 1995; Vuilleumier and Rafal, 2000; Rafal et al., 2002, 2006; Ptak and Schnider, 2005; experiment 3), an effect known as 'repetition blindness'. Repetition blindness occurs when subjects are presented with two identical target stimuli while their perceptual system's capacity is overwhelmed (e.g. by rapid and/or short duration presentation of stimuli). In this situation, performance for the second of the two target stimuli is typically impaired. While repetition blindness has mostly been demonstrated using stimuli that are separated temporally, it has also been shown to occur when two stimuli are presented simultaneously but separated spatially (Baylis et al., 1993; Kanwisher et al., 1995). Kanwisher (1987) has explained repetition blindness in the framework of the type-token hypothesis. This hypothesis differentiates between information regarding the identity of an object (type information) and information defining an object as a unique event (token information) and posits that only types that are bound to a token have access to perceptual awareness, where attention acts as the glue that binds a type to its token. This process of binding a type to its token is referred to as type individuation. According to this hypothesis, once an occurrence of a given type has been bound to a token, there is a time period wherein another occurrence of the same type can not be bound to a different token, i.e. the perceptual system can not generate two tokens in quick succession to a repeated presentation of the same type. Repetition blindness then occurs because the second presentation of the same type is not bound to a token. Repetition blindness has been associated with a changed ERP signal over posterior electrodes $200-400 \mathrm{~ms}$ from stimulus onset of the second target stimulus between trials where performance for the second of two identical targets is correct and trials where performance for the second of two identical targets is incorrect (Schendan et al., 1997; Koivisto and Revonsuo, 2008). There is however little agreement over the direction of change in the ERP signal. Nevertheless, the time course of the change in the ERP signal between trials where performance for the second of two identical targets is correct and trials where performance for the second of two identical targets is incorrect is consistent with accounts that repetition blindness reflects a failure of perceptual awareness occurring before access to working memory.
To summarize, stimulus similarity seems capable of eliciting opposite effects on task performance. Some studies offer evidence for the presence of similarity grouping while others put forward evidence for the presence of repetition blindness. However, these opposite effects of stimulus similarity on subject's performance have so far only been demonstrated in separate studies. This means that it can not be ruled out that these opposite findings reflect different strategies used by different subjects or elicited by different paradigms. In fact, the seminal study by Vuilleumier and Rafal (2000) performed on extinction patients already suggests that the nature of the experimental task can determine whether two identical target stimuli elicit similarity grouping or repetition blindness: when stimuli do not have to be identified, but instead only to be localized, two identical stimuli elicit similarity grouping whereas the identification of two identical stimuli results in repetition blindness. This suggests that the opposite effects of stimulus similarity on performance accuracy in previous studies can, at least in part, be explained by the different experimental paradigms used in these studies. It is however, so far unknown whether stimulus similarity has different effects on visual processing depending solely on the nature of the experimental task or whether both effects of stimulus similarity can coexist with either similarity grouping or repetition blindness dominating performance depending on the specific circumstances. A demonstration that similarity grouping and repetition blindness can coexist would help to determine more precisely the circumstances under which these opposite effects of stimulus similarity are elicited.

In the current manuscript we would like to posit that even in a single paradigm where subjects are instructed to always identify both stimuli it is possible to elicit both similarity grouping and repetition blindness by varying the amount of stimulus similarity. Specifically, based on suggestions by Duncan and Humphreys (1989), similarity grouping might not be limited to situations where the stimuli are identical but might also occur when the target stimuli are similar but not identical to one another. This prediction that target stimuli that are similar but not identical to one another can elicit similarity grouping has been supported by a study performed by Duncan (1989). Duncan studied reaction times in a visual search study where healthy subjects had to indicate whether or not a target, presented amongst irrelevant nontargets, was present. In the first experiment, he varied the similarity between the target and the nontargets and found that the reaction times to the target were longer the more similar the surrounding nontargets were to the target even if these nontargets were not identical to the target. Similar results were found by Pashler (1987). Moreover, in the second experiment Duncan varied the amount of similarity between the nontargets in the display, while keeping the similarity between the target and nontargets constant. He found that reaction times for the target stimulus were shorter when the nontargets were highly similar to one another as compared to when the nontargets were more dissimilar, even when the nontargets were not identical. Duncan explained these results by a combination of the effect of interalternative similarity and the effect of within-display similarity, where the effect of interalternative similarity is controlled by the classification of a stimulus as a possible target or nontarget and the effect of within-display similarity is controlled by perceptual grouping. Both interalternative similarity and within-display similarity 
increase target selection difficulty the more similar the target is to the nontargets and decrease target selection difficulty the more similar nontargets are to one another. Taken together, this study supports the prediction that even stimuli that are similar but not identical can theoretically elicit similarity grouping. On the other hand, target stimuli that are identical to one another should elicit both repetition blindness as well as similarity grouping. Crucially however, we hypothesize that similarity grouping and repetition blindness influence different aspects of stimulus processing. The type-token hypothesis differentiates between processing of type information and processing of information that individuates a stimulus as a unique event (token information). We hypothesize that similarity grouping can be seen as increasing the strength of the type information, whereas repetition blindness reflects a difficulty in rapidly generating multiple tokens to repeated occurrences of the same type. Thus, we expect that similarity grouping should dominate performance when the target stimuli are similar but not identical as these stimuli do not represent multiple occurrences of the same type and should therefore tend to generate unique tokens and no repetition blindness. On the other hand, when the target stimuli are identical, similarity grouping facilitates the processing of type information. However, performance will be impaired since due to repetition blindness this type information can not be correctly individuated, regardless of the strength of the type information. Thus, despite maximal similarity grouping, repetition blindness should dominate performance when the stimuli are identical.

A demonstration that repetition blindness and similarity grouping coexist and can both be elicited in the same subjects in a single task would not only help to determine more precisely the circumstances under which similarity grouping and repetition blindness are elicited, but would also provide a unique opportunity to directly compare both effects of stimulus similarity on task performance with respect to susceptibility to a modulating factor like attentional relevance. As will be described below, previous studies have provided inconclusive answers to whether the effect of stimulus similarity on task performance can be modulated by attentional relevance.

As mentioned, the type-token hypothesis which explains repetition blindness as an impairment to bind multiple occurrences of the same type to different tokens also postulates that attention plays a role in binding type information to token information (Kanwisher, 1987). More specifically, the binding of the first instance of a type to its token by attending to this type impairs the binding of the second instance of the same type to another token within a certain brief time period. This leads to the logical prediction that repetition blindness should depend critically on attention. To test this prediction, Kanwisher et al. (1995) presented neurologically healthy subjects with two simultaneously presented target stimuli where each target stimulus was one of three possible letters in one of three possible colours. The two target stimuli could be different from or identical to each other with respect to which letter and/or which colour and subjects were instructed to report either the letter or the colour. Their data suggested that if the subject was instructed to report the letters then repetition blindness only occurred for a repetition of the same letter whereas a repetition of the same colour had no effect on task performance. Conversely, if the subject was instructed to report the colours then repetition blindness only occurred for a repetition of the same colour whereas a repetition of the same letter had no effect on task performance. In other words, only repetition of a target stimulus on a relevant stimulus dimension led to repetition blindness. Similar effects of attentional relevance on repetition blindness have also been obtained in studies using neurological patients (Baylis et al., 1993). These findings suggest that attention is critical to the binding of type information to token information and have been taken as support for the typetoken hypothesis.

In contrast, the influence of attention on similarity grouping is less clear. Similarity grouping, like all principles of perceptual grouping, is generally thought to reflect an early pre-attentive process independent of selective attention (Treisman, 1982; Humphreys, 1998). This is supported by results that suggest that similarity grouping is associated with early neural activity in the occipital and temporal cortices (Han et al., 2002, 2005a,b). Moreover, neurological patients suffering from visual extinction generally display intact grouping effects (Mattingley et al., 1997; Vuilleumier and Rafal, 2000 experiment 1; Ward et al., 1994). Together, these studies strongly predict that attentional modulations should have no effect on similarity grouping.

Nevertheless, Mack et al. (1992) presented a series of studies which controversially suggest that attention does influence similarity grouping. They presented subjects with a briefly flashed cross which was superimposed on an irrelevant background of black and white squares. The subjects performed the difficult task of reporting which of the two lines making up the cross was longest, the horizontal or the vertical. Each subject was first presented with two trials where the black and white squares in the background were randomly distributed. In the third and critical trial, however, the black and white squares in the background were grouped on the basis of similarity. Each subject was subsequently asked to make a forced-choice decision on the nature of the similarity group in the background. Mack and colleagues showed that subjects were not able to perform better than chance on this forced-choice decision despite the fact that these subjects were able to perform both the line discrimination task and the similarity grouping task simultaneously after they had been made aware of the relevance of the background. These results suggest that similarity grouping does not occur when stimuli are not attended. However, Moore and Egeth (1997) convincingly argued that the results from Mack et al. (1992) were due to a failure to provide an overt response concerning the absence or presence of a similarity group under conditions of inattention and not an absence of similarity grouping per se. Their results suggested that similarity grouping does occur under conditions of inattention, but that the result of this grouping mechanism is not available for an overt response. A recent series of experiments performed by Lamy et al. (2006) confirmed these results.

Although these studies support the traditional idea that similarity grouping operates independently from selective attention, the results of several recent studies suggest that some Gestalt grouping mechanisms can be modulated by selective attention and that selective attention might modulate neural activity associated with grouping mechanisms in the primary visual area V1 (Freeman et al., 2001; Casco et al., 2005; Wu et al., 2005; Khoe et al., 2006). Yet these studies on whether selective attention can modulate Gestalt grouping mechanisms have traditionally studied 
the presence or absence of grouping effects for task irrelevant grouping stimuli presented either inside or outside the focus of spatial attention. Previous research however, suggests that repetition blindness can be modulated by the attentional relevance of an attribute of a stimulus presented within the focus of spatial attention (Baylis et al., 1993; Kanwisher et al., 1995); in these studies, only repetition of a stimulus on a relevant stimulus attribute led to repetition blindness, whereas repetition of a stimulus on an irrelevant stimulus attribute did not elicit repetition blindness. This means that to validly compare similarity grouping and repetition blindness with respect to susceptibility to a modulating factor like selective attention, we need to investigate whether similarity grouping can also be modulated by the attentional relevance of an attribute of a stimulus presented within the focus of spatial attention. Specifically, can similarity grouping be modulated by whether or not the similarity of target stimuli occurs on an attentionally relevant or an attentionally irrelevant stimulus attribute?

To our knowledge, only two studies, both from the same research group, aimed to answer this question. Han et al. (2005a,b) studied the influence of both spatial attention and attentional relevance on neural activation associated with similarity and proximity grouping. Whereas they found that stimuli presented outside the focus of spatial attention invariably did not elicit neural activation associated with either similarity or proximity grouping, their results on whether the attentional relevance of an attribute of a spatially attended stimulus can modulate Gestalt grouping mechanisms were inconsistent. The results of their fMRI study suggested that in the areas of the brain that mediate proximity grouping the neural activity associated with grouping was identical regardless of whether this grouping was relevant to the task at hand or not. On the other hand, the results of their ERP study suggested that the neural activity associated with both similarity and proximity grouping was suppressed when this grouping was attentionally irrelevant as compared to when this grouping was attentionally relevant.

In sum, previous studies have provided inconclusive answers to the question whether the opposite effects of stimulus similarity on task performance can be modulated by attentional relevance. Whereas there is considerable evidence that selective attention modulates repetition blindness, it is unclear whether attention should be expected to modulate similarity grouping or not. So far, however, no study has directly compared the effects of selective attention on both effects of stimulus similarity on task performance in a single experiment. The aim of this manuscript is then to directly compare both repetition blindness and similarity grouping with respect to their respective susceptibility to attentional relevance in the same subjects in a single design. Before this aim can be addressed however, the validity of the assumption that repetition blindness and similarity grouping coexist and can thus be elicited in a single experiment needs to be tested. The first experiment will thus test whether similarity grouping and repetition blindness can indeed both be elicited in the same experimental design. Once this has been established, the second experiment will subsequently directly compare the effects of attentional relevance on similarity grouping and repetition blindness in the same subjects in a single design.

\section{EXPERIMENT 1}

The aim of this experiment is to test whether similarity grouping and repetition blindness can be elicited in the same experimental design. By manipulating the degree of stimulus similarity between trials (with pairs of target stimuli being similar, different or identical), the aim is to establish that the opposite effects of stimulus similarity on perceptual performance reported in the literature do not reflect a strategic bias of particular individuals on specific paradigms. Based on previous findings we expect that similarity grouping will be elicited with perceptually similar but not identical target stimuli (with better performance for similar pairs than for different pairs of target stimuli). On the other hand, when the target stimuli are identical to one another, repetition blindness will be expected to dominate performance (with worse performance for identical pairs relative to different pairs of target stimuli).

\section{MATERIALS AND METHODS Subjects}

Twenty-four subjects ( 7 male, 17 female; mean age 21.75 years, range 20-31 years) participated in this study. Subjects were recruited from the student population of the University of Nottingham in the United Kingdom. All subjects reported being healthy with no history of neurological disorders and had normal or corrected to normal vision. All subjects were volunteers and signed an informed consent approved by the research ethics committee of the University of Nottingham.

\section{Experimental procedures}

The experiment was run on a computer with an AMD Athlon processor, running the Windows operating system. The experimental software Presentation (Neurobehavioral Systems Inc.) was used to present the stimuli. The monitor was a 19 inch (18 inch viewable) cathode ray tube Samsung Syncmaster 957P with a refresh rate of $100 \mathrm{~Hz}$ and a monitor resolution of $800 \times 600$ pixels. Subjects were seated in front of a computer screen at a distance of $57 \mathrm{~cm}$ with a chin rest used to maintain viewing distance. The background colour of the computer screen was always black. Each trial started with a $500 \mathrm{~ms}$ presentation of a centrally presented white fixation cross, straddled by two mask stimuli, one in each visual field. Each mask stimulus consisted of four grey (RGB 60,60,60) overlapping letters, namely 'O', 'C', 'E' and 'F' printed in the font 'Arial' at a font size of $40 \mathrm{pt}$. All letters were presented on the horizontal midline at an eccentricity of $8^{\circ}$ of visual angle from the central fixation cross. Subjects were instructed to fixate on the central fixation cross. Subsequently, a target display was overlaid on top of the mask stimuli. The presentation duration of the target display was determined for each subject individually with the aid of a staircase procedure in a separate titration experiment preceding the main experiment which aimed to reduce performance accuracy to $75 \%$. This was done to ensure that subjects were not at ceiling performance $(100 \%$ correct). Finally, the fixation cross and the mask stimuli were again presented during which the subject entered their responses. The next trial started $500 \mathrm{~ms}$ after the subject finished responding.

The target display consisted of unilateral or bilateral presentation of a green coloured letter which could be 'O', 'C', 'E' or ' $\mathrm{F}$ ' and subjects were instructed to identify the presented letter (during unilateral trials) or letters (during bilateral trials). In unilateral 
trials the target letter could be either low salience (roughly equal luminance to the mask stimulus: RGB $0,76,0$ ) or high salience (maximally salient green: RGB 0,255,0). Furthermore, the target letter could be presented in the left or the right visual field. Bilateral trials always consisted of a high salience and a low salience target letter, one in each visual field presented in the location of the two mask stimuli. Furthermore, in bilateral trials the two target letters could be different ('O' and 'E' ' $O$ ' and 'F', 'C' and ' $E$ ' or 'C' and ' $F$ '), similar (' $\mathrm{O}$ ' and ' $\mathrm{C}$ ' or ' $\mathrm{E}$ ' and ' $\mathrm{F}$ ') or same (' $\mathrm{O}$ ' and ' $\mathrm{O}$ ', ' $\mathrm{C}$ ' and ' $\mathrm{C}$ ', ' $E$ ' and ' $E$ ' or ' $F$ ' and ' $F$ '). These three types of bilateral trials were presented with equal frequency.

Similarity between the two target letters was defined by the amount of features both targets share, in line with the idea that the similarity between two letters is strongly correlated with the amount of contour that they share. Whereas the letters ' $\mathrm{E}$ ' and ' $\mathrm{F}$ ' and the letters ' $\mathrm{O}$ ' and ' $\mathrm{C}$ ' share many features the letters ' $\mathrm{E}$ ' and 'O', 'E' and 'C', 'F' and ' $O$ ' and ' $F$ ' and ' $C$ ' do not share features. Empirical evidence that supports the definition of similarity in this experiment was obtained from a so-called interconfusability matrix (van der Heijden et al., 1984). According to this matrix, the probabilities of $\mathrm{E}, \mathrm{F}, \mathrm{C}$ and $\mathrm{O}$ being reported as $\mathrm{E}$ is $0.350,0.057$, 0.049 and 0.002 respectively, the probabilities of $\mathrm{E}, \mathrm{F}, \mathrm{C}$ and $\mathrm{O}$ being reported as $\mathrm{F}$ is $0.183,0.367,0.014$ and 0.004 respectively, the probabilities of E, F. C and $\mathrm{O}$ being reported as $\mathrm{C}$ is $0.022,0.011$, 0.712 and 0.098 respectively and the probabilities of $\mathrm{E}, \mathrm{F}$. C and $\mathrm{O}$ being reported as $\mathrm{O}$ is $0.007,0.003,0.017$ and 0.383 respectively. In other words, the letters $\mathrm{E}$ and $\mathrm{F}$ and the letters $\mathrm{C}$ and $\mathrm{O}$ are systematically far more often confused with one another (which indicates high amounts of similarity) than the letters $\mathrm{E}$ and $\mathrm{C}, \mathrm{E}$ and $\mathrm{O}, \mathrm{F}$ and $\mathrm{C}$ and $\mathrm{F}$ and $\mathrm{O}$.

The rationale for combining high salience and low salience target letters in bilateral trials was that the bottom-up bias in salience should bias the competition for processing resources in favour of the high salience target stimuli. We predicted that this methodological manipulation would maximize the amount of trials in which at least the high salience target was perceived and thus would maximize both the amount of trials that can elicit repetition blindness (since repetition blindness is only elicited when at least one of the targets is identified) and the amount of trials that elicit similarity grouping (since the high salience target might function as an 'anchor' to which the low salience target can be grouped). Since this manipulation was expected to result in virtually maximum performance accuracy for the high salience target, we expected that the effects of similarity grouping and repetition blindness would be most apparent in performance accuracy for the low salience target stimuli. All subsequent statistical analyses therefore focus on these low salience target stimuli.

Subjects responded using two button boxes, on for each hand, which provided one response key for each finger. Subjects used the left hand button box for stimuli presented in the left visual field and the right hand button box for stimuli presented in the right visual field. Each target letter was associated with a finger ('O' with little finger, ' $C$ ' with ring finger, ' $F$ ' with middle finger and ' $E$ ' with index finger) and the button on the location of the thumb was used to indicate that there was no target presented in that visual field. Subjects were instructed to only respond with 'no target present' when they were confident that they did not see a target. They were told to guess if they saw a target letter, but were not sure about its identity. Subjects thus always had to provide two responses (one for each visual field) but were free to decide for themselves on the order in which they responded.

The experiment consisted of five blocks, with each block containing 84 trials. Specifically, each block consisted of 32 unilateral trials and 48 bilateral trials. Unilateral trials were included to allow a post-hoc check of the effectiveness of the titration experiment in ensuring none of the subjects was at ceiling performance during the main experiment. The order in which the trials were presented was randomized and each trial type appeared equally often. Therefore, per block 16 bilateral different, 16 bilateral similar and 16 bilateral same trials were presented. Finally, each block contained 4 catch trials where no stimuli were presented. Response accuracy was collected for each target stimulus.

The stimuli used in the titration experiment preceding the main experiment were identical to the stimuli used in the main experiment, except for the fact that in the titration experiment only unilaterally presented low salience target stimuli were used. Subjects were told that in each trial a single target stimulus was present and were instructed to either press the appropriate button on the left hand button box when they saw a target stimulus on the left or to press the appropriate button on the right hand button box when they saw a target stimulus on the right. They were told to always give a response and instructed to guess when they were unsure about the presence or identity of the target stimulus. The initial target presentation duration was $250 \mathrm{~ms}$ which was step-wise reduced by $10 \mathrm{~ms}$ when subject performance accuracy was higher than $75 \%$ in the preceding eight trials. The titration experiment ended when subject performance accuracy was equal to or lower than $75 \%$ in the preceding two sets of eight trials.

\section{RESULTS}

Trials with more than two responses (more than one per target stimulus) were excluded from analysis. Additionally, subjects whose performance showed either floor effects (performance on the low salience target stimuli not statistically different from chance) or ceiling effects (performance accuracy for the low salience target stimuli approaches $100 \%$ ) were excluded from further analyses. Consequently, a single subject (male, 20 years old) was excluded from statistical analyses because this subject's performance accuracy showed floor effects. The mean target presentation duration for the remaining 23 subjects was $200.43 \mathrm{~ms}$ (range 40-240 ms).

The percentage response accuracy scores were arcsin transformed before statistical analyses to normalise the distribution of the data (using the Microsoft Excel function “ $=\left\{2^{\star}\right.$ ASIN[POWER(value, $0.5)]\} / \mathrm{PI}()$ ", where 'value' is in the range $0 \ldots 1)$. The transformed mean performance accuracy for a unilaterally presented high salience target stimulus was $86.35 \%$ with a standard deviation of 6.39 while the mean performance accuracy for a unilaterally presented low salience target stimulus was $70.71 \%$ with a standard deviation of 8.95 . The transformed mean performance accuracy for the catch trials was $97.28 \%$ with a standard deviation of 4.28 . The transformed mean performance accuracies and the normalized standard deviations for each of the bilateral conditions for both the high salience and the low salience target stimuli are shown in Table 1 (see Loftus and Masson, 1994 for a discussion of why normalized 
Table 1 | Mean accuracy (\%) and normalized standard deviation (in brackets) for each bilateral condition for both the high salience and the low salience target stimulus.

\begin{tabular}{llll}
\hline \multicolumn{4}{c}{ Condition } \\
\hline Target type & bilateral similar & bilateral different & bilateral same \\
\hline High salience & $83.27(3.18)$ & $81.80(2.00)$ & $82.36(2.53)$ \\
Low salience & $68.85(3.24)$ & $63.58(3.42)$ & $59.07(4.78)$
\end{tabular}

values give a more accurate measure of the variability in a repeated measures design). Furthermore, the transformed mean performance accuracies for the low salience target stimuli for each of the bilateral conditions are shown in Figure 1.

The effect of similarity grouping on performance accuracy was assessed by statistically comparing performance accuracy during bilateral similar trials to performance accuracy during bilateral different trials. This paired sample $t$-test showed that performance accuracy during bilateral similar trials was significantly higher than performance accuracy during bilateral different trials $(t=5.445$, $\mathrm{df}=22, p<0.001)$.

The effect of repetition blindness on performance accuracy was assessed by statistically comparing performance accuracy during bilateral same trials to performance accuracy during bilateral different trials. This paired sample $t$-test showed that performance accuracy during bilateral same trials was significantly lower than performance accuracy during bilateral different trials $(t=2.825$, $\mathrm{df}=22, p=0.01)$.

The results did not fundamentally differ when all statistical analyses were performed on the original not arcsin transformed accuracy scores.

To assess whether the effect of repetition blindness might be due to a response bias against giving the same response with both hands an additionally analysis was performed on the types of errors made in the bilateral conditions. Errors were categorized as 'perceived similar errors' (for example, responding ' $E$ ' and ' $F$ ' when an 'E' and an 'O' were presented), 'perceived different errors' (for example, responding ' $E$ ' and ' $C$ ' when an ' $E$ ' and an ' $O$ ' were presented), 'perceived same errors' (for example, responding ' $E$ ' and 'E' when an 'E' and an 'O' were presented) or 'perceived unilateral errors' (for example responding ' $E$ ' and 'nothing' when an 'E' and an 'O' were presented). This analysis showed that in the bilateral similar condition, $8 \%$ of the errors were 'perceived similar errors', $41 \%$ were 'perceived different errors', $39 \%$ were 'perceived same errors' and $11 \%$ were 'perceived unilateral errors'. In the bilateral different condition, $24 \%$ of the errors were 'perceived similar errors', $53 \%$ were 'perceived different errors', $13 \%$ were 'perceived same errors' and $10 \%$ were 'perceived unilateral errors'. Finally, in the bilateral same condition, $57 \%$ of the errors were 'perceived similar errors', $28 \%$ were 'perceived different errors', $6 \%$ of the errors were 'perceived same errors' and $8 \%$ were 'perceived unilateral errors'. The results of this analysis suggest that, particularly in the bilateral similar condition, subjects did not have a problem giving the same response with both hands. Instead, these results suggest that subjects tended to mistake two similar stimuli for two identical stimuli and vice versa.

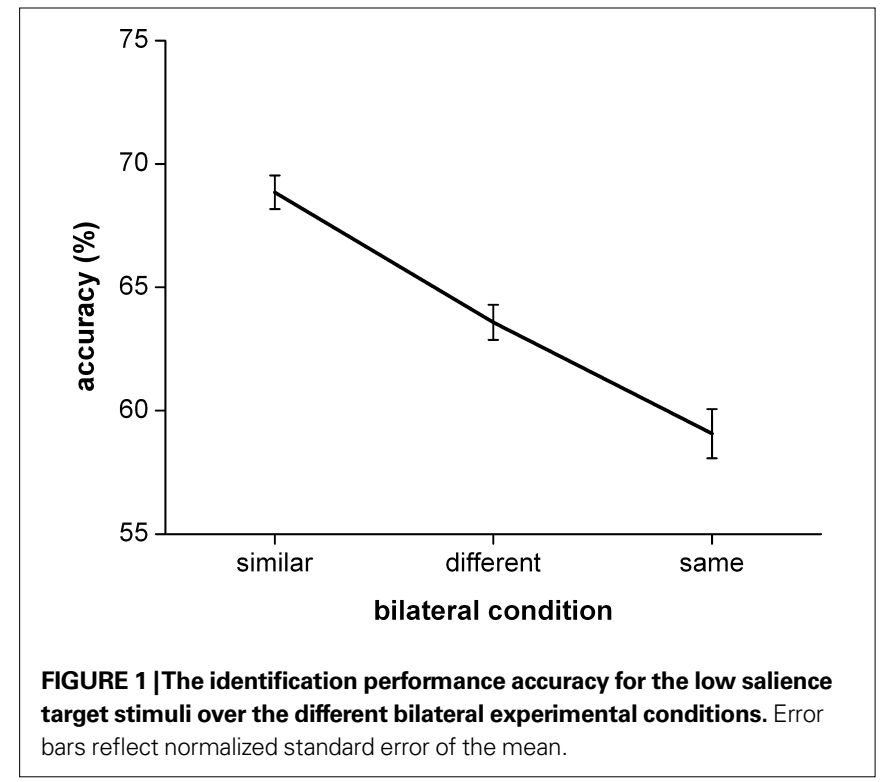

\section{DISCUSSION}

The results from experiment 1 suggest that both similarity grouping and repetition blindness can be elicited in a single experimental design. During bilateral trials, relative to performance accuracy when the two target stimuli were different, similarity of the two target stimuli led to an increase in performance accuracy and identicality of the two target stimuli led to a decrease in performance accuracy. These findings are in line with the results from previous studies that found that two identical target stimuli that need to be identified give rise to repetition blindness (Baylis et al., 1993; Kanwisher et al., 1995; Vuilleumier and Rafal, 2000; Rafal et al., 2002, 2006; Ptak and Schnider, 2005, experiment 3). These findings also confirm the hypothesis that similarity grouping is not confined to situations were the multiple targets are identical (as was the case in the studies by Vuilleumier and Rafal (2000, experiment 1) and Ward et al. (1994), but can also be elicited when targets are similar to one another (Pashler, 1987; Duncan, 1989; Duncan and Humphreys, 1989). Moreover, while similarity grouping of multiple targets has previously been demonstrated in neurological patients suffering from the attentional deficit known as extinction, these results suggest that this effect can also be elicited in neurologically healthy subjects. This provides support for the proposal that biased competitive interactions underlie visual perception in neurologically healthy subjects and that extinction can be seen as an exaggeration of normal behaviour (Desimone and Duncan, 1995; Duncan et al., 1997; Desimone, 1998; Duncan, 1998). Finally, unlike previous studies, the present findings reveal both repetition blindness and similarity grouping in the same experiment (across interleaved trials), demonstrating that these two effects can coexist and do not simply reflect different global strategies used by different individuals (or elicited by different paradigms).

Since the results of Experiment 1 confirm that both similarity grouping and repetition blindness can be elicited in a single experimental design, the aim of this manuscript can be addressed; can both these effects of stimulus similarity on task performance be modulated by attentional relevance? This question 
will be investigated in the second experiment by comparing the effects of attentional relevance on similarity grouping and repetition blindness.

\section{EXPERIMENT 2}

This second experiment directly compares the opposite effects of stimulus similarity on task performance with respect to their respective susceptibility to attentional relevance in a single design in the same subjects. Based on the previous literature, it is expected that repetition blindness critically depends on attention. In other words, repetition blindness should only occur when the target stimuli are identical on a task relevant attribute. However, whether similarity grouping can be modulated by attentional relevance is uncertain. Specifically, this experiment could reveal that similarity grouping occurs when the target stimuli are similar with respect to either the attentionally relevant or the attentionally irrelevant attribute (not modulated by attentional relevance) or only occurs when the target stimuli are similar with respect to the attentionally relevant attribute (modulated by attentional relevance).

\section{MATERIALS AND METHODS \\ Subjects}

Twenty-one subjects ( 6 male, 14 female; mean age 21.05 years, range 19-25 years; age and sex of 1 subject not recorded) participated in this study. Subjects were recruited from the local student population of the University of Nottingham in the United Kingdom. All subjects reported being healthy with no history of neurological disorders and had normal or corrected to normal vision. All subjects were volunteers and signed an informed consent approved by the research ethics committee of the University of Nottingham.

\section{Experimental procedures}

The experimental procedures were the same as in Experiment 1 of this manuscript. However, unlike the first experiment, the target stimuli were now presented in four possible colours. As in Experiment 1, the different colours could be either low salience (roughly equal luminance with the mask stimulus which was grey RGB 40,40,40) or high salience (roughly equal luminance with maximum salience red which was RGB 255,0,0). The four possible colours were green (low salience RGB 0,55,0; high salience RGB $0,135,0$ ), turquoise (low salience RGB 0,50,40; high salience RGB $0,135,80$ ), magenta (low salience RGB 100,0,40; high salience RGB $235,0,80$ ) and red (low salience RGB 105,0,0; high salience RGB $255,0,0)$. The colour we refer to as turquoise was created by adding a small amount of blue to the colour green and the colour we refer to as magenta was created by adding a small amount of blue to the colour red. These four colours and their names were shown to the subjects before the experiment started and subjects were allowed to inspect them as long as they wanted. To summarise, each target stimulus consisted of one out of four possible letters printed in one out of four possible colours.

The subjects had to perform two tasks: letter identification or colour identification. During the letter identification task, subjects were again instructed to identify the letters ('O', 'C', ${ }^{\prime} F^{\prime}$ or ' $E$ '). During the colour identification task subjects were instructed to identify the colours (green, turquoise, magenta or red). The order in which the two tasks were presented was randomized for each subject. As in
Experiment 1, in bilateral trials the letters could be different, similar or same. However, in this experiment, additionally the colours in which the letters were printed could also be different (green + red, green + magenta, turquoise + red or turquoise + magenta), similar (green + turquoise or red + magenta) or same (green + green, turquoise + turquoise, red + red or magenta + magenta). Combined this meant that in bilateral trials the target stimuli could be different, similar or same on both the attentionally relevant stimulus attribute (letter in the letter identification task and colour in the colour identification task) and the attentionally irrelevant stimulus attribute (letter in the colour identification task and colour in the letter identification task).

In the letter identification task, stimulus similarity was defined as in Experiment 1. In the colour identification task, stimulus similarity was defined based on the standard geometrical representation of colour space as a circle red-orange-yellow-green-blue-purplered, analogous to the definition of colour similarity adopted by Duncan (1989). According to this colour space, the colours green and turquoise (created by adding a small amount of blue to the colour green) and the colours red and magenta (created by adding a small amount of blue to the colour red) are more similar to each other than the colours green and magenta, green and red, turquoise and magenta and turquoise and red.

Each task consisted of five blocks of 90 trials. Each task contained 100 unilateral trials with each possible trial type occurring with equal likelihood and 345 bilateral trials with each possible trial type occurring with equal likelihood. For both the relevant and the irrelevant atttentional dimension, 115 of the bilateral trials consisted of two different target stimuli, 115 trials consisted of two similar target stimuli and 115 trials consisted of two identical target stimuli. Finally, 5 catch trials were presented.

Subjects responded using the same two button boxes as in Experiment 1. Each target letter was associated with a finger. For the letter identification task the same fingers as in Experiment 1 were used to respond; ' $O$ ' with little finger, ' $\mathrm{C}$ ' with ring finger, ' $\mathrm{F}$ ' with middle finger and ' $\mathrm{E}$ ' with index finger. For the colour identification task the responses were green with little finger, turquoise with ring finger, red with middle finger and magenta with index finger. The button on the location of the thumb was used to indicate that there was no target presented in that visual field.

For both tasks, the presentation duration of the target display was determined for each subject individually with the aid of a staircase procedure in separate titration experiments preceding the main experiments to ensure that subjects were not at ceiling performance. The titration experiments were identical to the titration experiment of Experiment 1, except for the fact that subjects were instructed to identify the letter in the titration experiment preceding the letter identification task and to identify the colour in the titration experiment preceding the colour identification task. The initial target presentation duration was $250 \mathrm{~ms}$ which was step-wise reduced by $10 \mathrm{~ms}$ when subject performance accuracy was higher than $70 \%$ in the preceding 10 trials. The titration experiment ended when subject performance accuracy was equal to or lower than $70 \%$ in the preceding two sets of 10 trials.

Response accuracy was collected for each target stimulus and, as in Experiment 1, only the performance on the low salience stimuli was statistically analyzed. 


\section{RESULTS}

Trials with more than one response per target stimulus were excluded from analysis. Additionally, subjects whose performance showed either floor or ceiling effects were excluded from further analyses. Consequently, two subjects (both male, 20 and 25-years old) were excluded from statistical analyses of the colour identification task because their performance accuracy showed floor effects (performance not significantly different from chance). This left 21 subjects for the statistical analyses of the letter identification task and 19 subjects for the statistical analyses of the colour identification task. The mean target presentation duration in the letter identification task was $184.76 \mathrm{~ms}$ (range 20-240 ms) and the mean target presentation duration in the colour identification task was $51.58 \mathrm{~ms}$ (range 10-230 ms).

The percentage response accuracy scores were arcsin transformed before statistical analysis. In the letter identification task, the transformed mean performance accuracy for a unilaterally presented high salience target stimulus was $86.22 \%$ with a standard deviation of 12.36 while the transformed mean performance accuracy for a unilaterally presented low salience target stimulus was $66.79 \%$ with a standard deviation of 9.02 . The transformed mean performance accuracy for the catch trials was $72.63 \%$ with a standard deviation of 34.22 . In the colour identification task, the transformed mean performance accuracy for a unilaterally presented high salience target stimulus was $81.48 \%$ with a standard deviation of 11.24 while the transformed mean performance accuracy for a unilaterally presented low salience target stimulus was $58.38 \%$ with a standard deviation of 12.56 . The transformed mean performance accuracy for the catch trials was $71.77 \%$ with a standard deviation of 29.36. The lower catch trial performance accuracy in this experiment when compared to the catch trial performance in Experiment 1 can most likely be explained by the fact that in this experiment only $1 \%$ of all trials were catch trials whereas $5 \%$ of all trials were catch trials in Experiment 1.

The transformed mean performance accuracies and normalized standard deviations for each of the bilateral conditions for both the high salient and the low salient target stimuli are shown in Table 2. Furthermore, the transformed mean performance accuracies for the low salient target stimuli for each of the bilateral conditions are shown in Figure 2.

To assess the effect of attentional relevance on similarity grouping, a repeated measures 2 (relevant stimulus attribute condition: bilateral similar or bilateral different) by 2 (irrelevant stimulus attribute condition: bilateral similar or bilateral different) ANOVA was performed for both the colour identification task and the letter identification task. This ANOVA showed a significant main effect for the attentionally relevant stimulus attribute condition in the letter identification task $\left(F_{1,20}=24.906, p<0.001\right)$ whereas both the main effect of the attentionally irrelevant stimulus attribute condition and the interaction effect failed to reach significance $\left(F_{1,20}=0.141, p=0.711\right.$ and $F_{1,20}=0.569, p=0.460$ respectively). Stimulus similarity on the relevant stimulus attribute significantly improved performance accuracy in the letter identification task, whereas stimulus similarity on the irrelevant stimulus attribute had no effect on performance accuracy. For the colour identification task, this ANOVA showed no significant main effects or interaction effect $\left(F_{1,18}=1.116, p=0.305\right.$ for the relevant stimulus attribute effect, $F_{1,18}=0.469, p=0.502$ for the irrelevant stimulus attribute effect and $F_{1,18}=0.207, p=0.654$ for the interaction effect). In other words, in the colour identification task, stimulus similarity on neither the relevant nor the irrelevant stimulus attribute had a significant effect on performance accuracy.

Likewise, to assess the effect of attentional relevance on repetition blindness, a repeated measures 2 (relevant stimulus attribute: bilateral same or bilateral different) by 2 (irrelevant stimulus attribute: bilateral same or bilateral different) was performed for both the colour identification task and the letter identification task. This ANOVA showed no significant main effect or interaction effect for the letter identification task $\left(F_{1,20}=2.158, p=0.157\right.$ for the relevant stimulus attribute effect, $F_{1,20}=1.676, p=0.210$ for the irrelevant stimulus attribute effect and $F_{1,20}=1.233, p=0.280$ for the interaction effect). In other words, in the letter identification task, stimulus identicality on neither the relevant nor the irrelevant stimulus attribute had a significant effect on performance accuracy. For the colour identification task, this ANOVA showed a significant main effect for the attentionally relevant stimulus attribute condition $\left(F_{1,18}=17.453\right.$, $p=0.001$, whereas both the main effect for the attentionally irrelevant stimulus attribute condition and the interaction effect failed to reach significance $\left(F_{1,18}=0.282, p=0.602\right.$ and $F_{1,18}=0.271, p=0.609$ respectively). In the colour identification task, stimulus identicality on the relevant stimulus attribute significantly decreased performance accuracy while stimulus identicality on the irrelevant stimulus attribute had no significant effect on performance accuracy.

The results did not fundamentally differ when all statistical analyses were performed on the original not arcsin transformed accuracy scores.

Table 2 | Mean accuracy (\%) and normalized standard deviations (in brackets) for each of the bilateral conditions for both the high salience and the low salience target stimulus in both the letter identification and the colour identification task.

\begin{tabular}{|c|c|c|c|c|c|c|c|c|c|}
\hline \multirow{2}{*}{$\begin{array}{l}\text { Relevant attribute: } \\
\text { Irrelevant attribute: }\end{array}$} & \multicolumn{3}{|c|}{ Similar } & \multicolumn{3}{|c|}{ Different } & \multicolumn{3}{|c|}{ Same } \\
\hline & Similar & Different & Same & Similar & Different & Same & Similar & Different & Same \\
\hline High salience targets & 84.88 (5.49) & $81.26(7.00)$ & $84.23(4.43)$ & $83.29(6.91)$ & 81.35 (5.23) & $82.59(4.07)$ & $82.52(6.01)$ & $85.87(7.78)$ & $82.55(6.08)$ \\
\hline Low salience targets & $63.20(4.98)$ & $62.87(5.22)$ & $61.25(6.01)$ & $57.06(5.56)$ & $58.31(4.22)$ & $58.37(5.89)$ & $55.01(5.01)$ & $54.75(4.93)$ & $57.39(6.75)$ \\
\hline Low salience targets & $47.98(5.30)$ & $48.10(5.99)$ & $48.71(6.72)$ & $46.67(5.25)$ & $49.87(4.71)$ & $49.99(5.00)$ & $40.21(6.23)$ & $40.39(8.62)$ & $41.55(6.55)$ \\
\hline
\end{tabular}



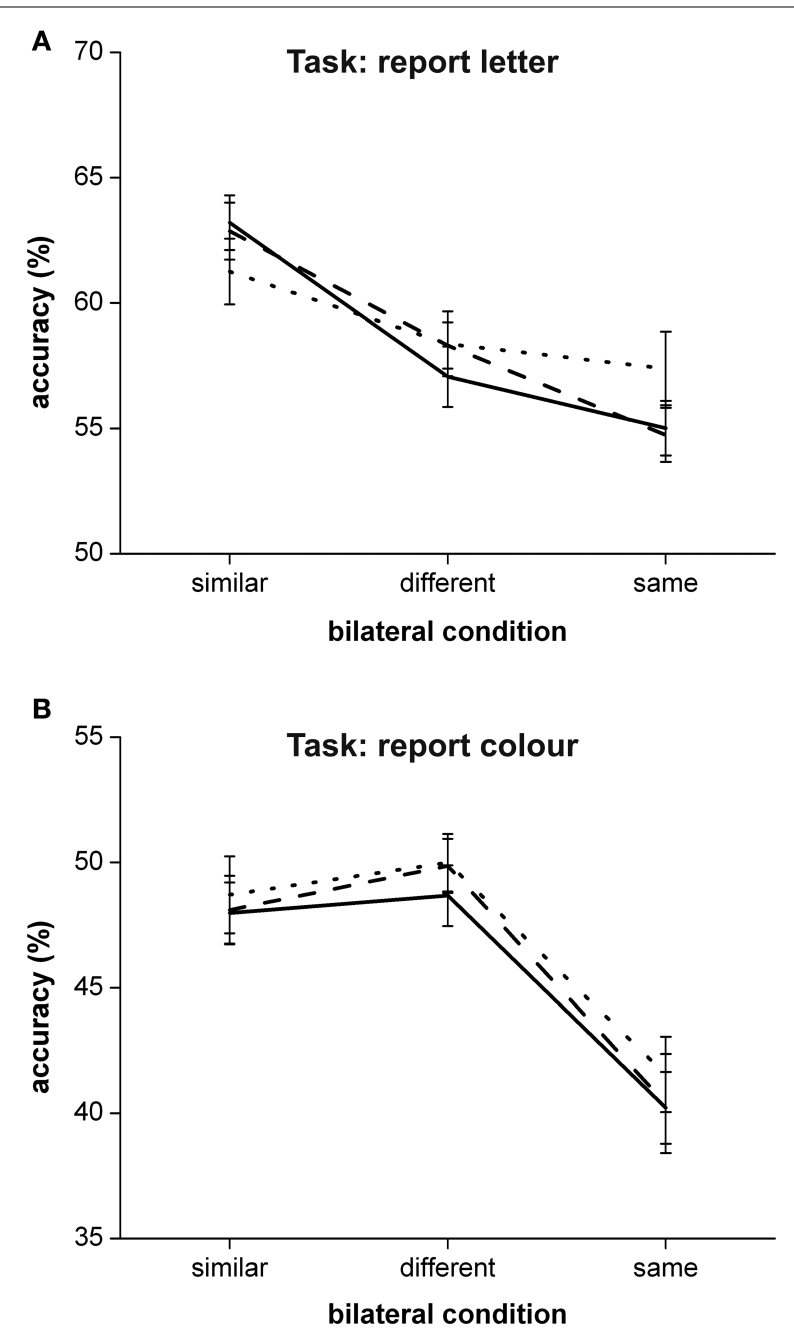

FIGURE 2 |The identification performance accuracy for the low salience target stimuli over the different bilateral experimental conditions in the letter identification task (A) and the colour identification task (B). The relevant stimulus attribute condition is plotted along the $x$-axis. The different lines reflect the different irrelevant stimulus attribute conditions; solid line $=$ bilateral similar, striped line $=$ bilateral different, dotted line $=$ bilateral same. Error bars reflect normalized standard error of the mean.

As in Experiment 1, to assess whether the effect of repetition blindness might be due to a response bias against giving the same response with both hands an additionally analysis was performed on the types of errors made in the bilateral conditions of both the letter identification task and the colour identification task. Errors were again categorized as 'perceived similar errors', 'perceived different errors', 'perceived same errors' or 'perceived unilateral errors'. The results of this analysis showed that the types of errors made in the bilateral conditions of Experiment 2 (averaged over all irrelevant conditions) showed an identical pattern to the types of errors made in the bilateral conditions of experiment 1 . In the bilateral similar condition of the letter identification task, $6 \%$ of the errors were 'perceived similar errors', $26 \%$ were 'perceived different errors', 28\% were 'perceived same errors' and 40\% were 'perceived unilateral errors'. In the bilateral different condition of the letter identification task, $18 \%$ of the errors were 'perceived similar errors', $35 \%$ were 'perceived different errors', $11 \%$ were 'perceived same errors' and $35 \%$ were 'perceived unilateral errors'. In the bilateral same condition of the letter identification task, $37 \%$ of the errors were 'perceived similar errors', $22 \%$ were 'perceived different errors', $6 \%$ were 'perceived same errors' and $34 \%$ were 'perceived unilateral errors'. In the bilateral similar condition of the colour identification task, $3 \%$ of the errors were 'perceived similar errors', $8 \%$ were 'perceived different errors', $15 \%$ were 'perceived same errors' and $73 \%$ were 'perceived unilateral errors'. In the bilateral different condition of the colour identification task, $6 \%$ of the errors were 'perceived similar errors', $21 \%$ were 'perceived different errors', $5 \%$ were 'perceived same errors and $68 \%$ were 'perceived unilateral errors. Finally, in the bilateral same condition of the colour identification task, $23 \%$ of the errors were 'perceived similar errors', $4 \%$ were 'perceived different errors', 9\% were 'perceived same errors' and $63 \%$ were 'perceived unilateral errors'. In other words, as in Experiment 1, the results of this analysis suggest that, particularly in the bilateral similar conditions, subjects did not have a problem giving the same response with both hands. Instead, these results again suggest that subjects tended to mistake two similar stimuli for two identical stimuli and vice versa.

\section{DISCUSSION}

The results from Experiment 2 suggest that both effects of stimulus similarity on task performance can be modulated by selective attention. More precisely, these results suggest that both similarity grouping and repetition blindness can be influenced by attentional relevance of spatially attended stimulus attributes. When similarity grouping or repetition blindness occurred, it was restricted to the attentionally relevant stimulus attribute and eliminated when the stimulus attribute was attentionally irrelevant. When subjects had to identify the colours, stimulus identicality elicited repetition blindness only when the stimulus attribute was relevant and not when the stimulus attribute was irrelevant. This is in line with previous studies in both healthy subjects and neurological patients that suggest that repetition blindness depends on attention (Baylis et al., 1993; Kanwisher et al., 1995). When subjects had to identify the letters, stimulus similarity likewise elicited similarity grouping only when the stimulus attribute was relevant and not when the stimulus attribute was irrelevant. This suggests that, like repetition blindness, similarity grouping also depends on attention. This novel finding supports controversial results from a growing body of studies that suggest that perceptual grouping mechanisms thought to operate pre-attentively might under certain conditions not be as immune to attentional modulations as generally thought (Mack et al., 1992; Freeman et al., 2001; Casco et al., 2005; Han et al., 2005b; Wu et al., 2005; Khoe et al., 2006).

However, the results also suggest that repetition blindness could not be elicited for the relevant stimulus attribute in the letter identification task and that similarity grouping did not occur for the relevant stimulus attribute in the colour identification task. In the letter identification task, performance accuracy did decrease when the two stimuli were identical on the relevant stimulus attribute, suggesting that some repetition blindness did occur. However, this decrease in performance accuracy failed to reach significance when compared with performance accuracy for two different stimuli $(p=0.157)$. 
Nevertheless, while there was a trend towards evidence of repetition blindness for the attentionally relevant stimulus attribute, for the attentionally irrelevant stimulus attribute, if anything, performance seemed to be better when the stimuli were identical as compared to when they were different. Thus, combined with the results from Experiment 1, which showed a highly significant effect of repetition blindness during an essentially identical letter identification task, the data do not necessarily contradict the notion that in the letter identification task repetition blindness depends on attentional relevance. The lack of a significant effect of repetition blindness however, does not allow a firm conclusion concerning whether repetition blindness can be modulated by attentional relevance in the letter identification task.

In the colour identification task, on the other hand, no evidence at all was found for an effect of similarity grouping on task performance. If anything, task performance accuracy slightly decreased when the two stimuli were similar on the relevant stimulus attribute when compared to performance accuracy for two different stimuli. So why did Experiment 2 fail to find evidence of similarity grouping in the colour identification task? Most likely it has something to do with the definition of stimulus similarity in this task. In the letter identification task, stimulus similarity was created by making a small change to one of two identical stimuli in line with the idea that the similarity between two letters is strongly correlated with the amount of contour that they share. A small part was removed from either an ' $E$ ' or an ' $\mathrm{O}$ ' creating an ' $\mathrm{F}$ ' (hypothesized to be similar to an 'E') or a ' $C$ ' (hypothesized to be similar to an ' $O$ '). This definition of stimulus similarity can be fairly objectively quantified and has been empirically supported by the interconfusability matrix for capital letters by van der Heijden et al. (1984). Furthermore, the presence of a significant increase in performance accuracy for two similar stimuli when compared to the performance accuracy for two different stimuli in Experiment 1 suggests that these stimuli are capable of eliciting similarity grouping. However, unlike the definition of stimulus similarity in the letter identification task, the definition of stimulus similarity in the colour identification task cannot be so objectively quantified. In the colour identification task, as in the letter identification task, creating stimulus similarity was attempted by making a small change to one of two identical stimuli. In this case, a small amount of blue was added to either red or green creating magenta (hypothesized to be similar to red) or turquoise (hypothesized to be similar to green) respectively. This definition of stimulus similarity was based on the standard geometrical representation of colour space as a circle red-orange-yellow-green-blue-purple-red, analogous to the definition of colour similarity adopted by Duncan (1989). However, it is highly possible that this attempt to create stimulus similarity in the colour identification task was not successful. As a consequence, no similarity grouping was elicited.

To summarize, the data of this experiment support the idea that the effect of stimulus similarity on perceptual performance depends on selective attention. Both similarity grouping and repetition blindness were modulated by attentional relevance. In the letter identification task, similarity grouping occurred only when the stimulus attribute was attentionally relevant and not when the stimulus attribute was attentionally irrelevant. Likewise, in the colour identification task, repetition blindness only occurred when the stimulus attribute was attentionally relevant and not if the stimulus attribute was attentionally irrelevant.

\section{GENERAL DISCUSSION}

The aim of this manuscript was to directly compare the two opposite effects of stimulus similarity on performance accuracy, namely repetition blindness and similarity grouping, with respect to their susceptibility to modulation by attentional relevance. This aim was based on the assumption that repetition blindness and similarity grouping coexist and can thus both be elicited in a single design in the same subjects, thereby allowing a direct comparison between these phenomena. Experiment 1 was designed to test the validity of this assumption. The results of this experiment suggest that repetition blindness and similarity grouping can be elicited in the same subjects in a single design. Whereas two identical target stimuli give rise to repetition blindness which elicits a decrease in identification performance accuracy, two target stimuli that are similar to one another (but not identical) elicit similarity grouping which results in an increase in identification performance accuracy. Therefore, these results replicate in a single paradigm seemingly paradoxical findings from previous studies (Baylis et al., 1993; Kanwisher et al., 1995; Vuilleumier and Rafal, 2000; Ptak and Schnider, 2005, experiment 3; Ward et al., 1994). These results thus confirm that repetition blindness and similarity grouping coexist and can be elicited in the same subjects in a single design. The finding that repetition blindness and similarity grouping can be elicited in a single design provided the opportunity to directly compare these opposite effects of stimulus similarity with respect to susceptibility to attentional relevance in Experiment 2.

The results from Experiment 2 firstly suggest that repetition blindness is influenced by attentional relevance. This finding is in line with the results from several previous studies (Baylis et al., 1993; Kanwisher et al., 1995). Kanwisher (1987) explains repetition blindness in the framework of the type-token hypothesis which differentiates between type information (information regarding the identity of an object) and token information (information defining an object as a unique event). The type-token hypothesis posits that repetition blindness occurs when multiple occurrences of the same type are not recognized as different tokens. Furthermore, this theory postulates that attention plays a role in linking type information to token information. The observation that repetition blindness is modulated by attentional relevance seems to support this hypothesis.

One of the largest criticisms that have been raised against the typetoken explanation of repetition blindness is that repetition blindness does not reflect a perceptual bias, but instead a response bias where subjects are simply biased against giving the same response with both hands. In our study, a subject bias against giving the same response with both hands could explain the observation that only the attentionally relevant stimulus dimension generates repetition blindness. However, the results of the analyses of the types of errors made in the bilateral conditions of both experiments strongly suggest that, particularly in the bilateral similar conditions, subjects did not have a problem giving the same response with both hands under conditions of uncertainty, which would have been expected if our subjects had a bias against giving two identical responses. Instead, our results 
suggest that subjects tended to mistake two similar stimuli for two identical stimuli and vice versa, which seems plausible considering that these bilateral conditions contained stimuli that are highly similar. Given that in the bilateral conditions errors resulting in the subject giving two different responses ('perceived similar errors' and 'perceived different errors') were three times as likely as errors resulting in the subject giving two identical responses, the amount of errors involving two identical responses in the bilateral similar conditions of both experiments was remarkably high (e.g. if the subject was required to identify the letter and for example perceive the high salience stimulus to be an 'E', three of the five possible responses for the low salience stimulus would have involved giving two different responses and only one of the five possible responses would have involved giving two identical responses). Moreover, whereas some studies suggest that repetition blindness can (partly) be explained by a response bias (Armstrong and Mewhort, 1995; Fagot and Pashler, 1995; Anderson and Neill, 2002) the majority of studies have tended to support the view of repetition blindness as a perceptual phenomenon that cannot be explained by a response bias (Park and Kanwisher, 1994; Kanwisher et al., 1996; Johnston et al., 2002; Morris and Harris, 2004). Most notably, ERP studies suggest that repetition blindness reflects a failure of perceptual awareness occurring before access to working memory (Schendan et al., 1997; Koivisto and Revonsuo, 2008). Taken together, we feel that this strongly argues against a response bias explanation of our repetition blindness effect.

Secondly, Experiment 2 reveals a novel finding: similarity grouping also depends on selective attention. Specifically, when the grouping stimuli were presented within the focus of spatial attention, Gestalt grouping mechanisms were modulated by whether this grouping was attentionally relevant or not. Similarity grouping, like all perceptual grouping mechanisms, is generally thought to reflect an early pre-attentive process independent of attention (Treisman, 1982; Humphreys, 1998). The present results however, seem to confirm the controversial suggestion that perceptual grouping mechanisms can be modulated by selective attention (Han et al., 2005b). In the present study subjects were not required to overtly report the nature of the similarity grouping. Subjects were instead merely required to identify one of two possible stimulus attributes for two target stimuli. In other words, in the present experiment, unlike in the experiment performed by Mack et al. (1992), the presence or absence of similarity grouping effects was indirectly measured, a mere failure to report the perceptual group can therefore not explain the present results.

The finding that early perceptual grouping mechanisms can be modulated by attentional relevance has clear theoretical implications. According to the theory of biased competitive interactions, the multiple neural representations in the brain associated with the stimuli in the visual field compete for limited processing resources (Desimone and Duncan, 1995; Duncan et al., 1997; Desimone, 1998; Duncan, 1998). However, before this competition between stimulus representations can take place, the stimulus representations themselves must be formed. The theory of biased competitive interactions suggests that the competition between the multiple stimulus representations is preceded by a pre-attentive parallel processing stage where the visual field is organized in stimulus groups (for a large part on the basis of Gestalt grouping principles) on which these competitive interactions can subsequently be performed. In other words, this theory predicts a dichotomy between pre-attentive mechanisms that organise the visual input in stimulus groups and attentive mechanisms that operate on those groups. However, the results of the present study which suggest that attentional relevance can modulate grouping mechanisms are incompatible with this dichotomous view.

The present observation that the attentional relevance of grouping stimuli presented within the focus of spatial attention can modulate perceptual grouping mechanisms is in line with suggestions based on animal research that perceptual grouping can be roughly divided in two stages: an early stage of feedforward processing and a later stage of recurrent processing (see Roelfsema, 2006 for a review). This proposal suggests that the early perceptual grouping stage associated with feedforward processing is pre-attentive, i.e. immune to attentional modulations whereas the later stage associated with recurrent processing can be modulated by top-down influences like attentional relevance. This idea that perceptual grouping mechanisms can be divided in two stages has, at least for proximity grouping, recently been confirmed by electrophysiological studies in humans (Nikolaev et al., 2008). Moreover, electrophysiological studies in humans have supported the suggestion that perceptual grouping mechanisms can be influenced via recurrent processing by spatial attention (Casco et al., 2005; Han et al., 2005a,b; Khoe et al., 2006) and attentional relevance of spatially attended grouping stimuli (Han et al., 2005b). The present results are thus compatible with the proposal that at least some aspects of perceptual grouping processes can be modulated by top-down attentional factors.

In conclusion, the present study suggests that repetition blindness and similarity grouping can both be modulated by attentional relevance. This confirms the predictions made by the type-token hypothesis and suggests that supposedly pre-attentive grouping mechanisms might not be as immune to top-down attentional modulations as traditionally thought.

\section{ACKNOWLEDGMENTS}

We would like to thank Ben Davis, Kathy Niu and the two reviewers for helpful comments on earlier versions of this manuscript. This research was supported by NIH grant R01 NS054266.

\section{REFERENCES}

Anderson, C. J., and Neill, W. T. (2002). Two Bs or not two Bs? A signal detection theory analysis of repetition blindness in a counting task. Percept. Psychophys. 64, 732-740.

Armstrong, I. T., and Mewhort, D. J. K. (1995). Repetition deficit in rapid-serial-visual-presentation displays: encoding failure or retrieval failure? J. Exp. Psychol. Hum. Percept. Perform. 21, 1044-1052.

Baylis, G. C., Driver, J., and Rafal, R. D. (1993). Visual extinction and stimulus repetition. J. Cogn. Neurosci. 5, 453-466.
Becker, E., and Karnath, H. O. (2007). Incidence of visual extinction after left versus right hemisphere stroke. Stroke 38, 3172-3174.

Casco, C., Grieco, A., Campana, G., Corvino, M. P., and Caputo, G. (2005). Attention modulates psychophysical and electrophysiological response to visual texture segmentation in humans. Vision Res. 45, 2384-2396.

Desimone, R. (1998). Visual attention mediated by biased competition in extrastriate visual cortex. Philos. Trans. R. Soc. Lond., B, Biol. Sci. 353, 1245-1255. 
Desimone, R., and Duncan, J. (1995). Neural mechanisms of selective visual attention. Annu. Rev. Neurosci. 18, 193-222.

Duncan, J. (1989). Boundary conditions on parallel processing in human vision. Perception 18, 457-469.

Duncan, J. (1998). Coverging levels of analysis in the cognitive neuroscience of visual attention. Philos. Trans. R. Soc. Lond., B, Biol. Sci. 353, 1307-1317.

Duncan, J., Humphreys, G., and Ward, R. (1997). Competitive brain activity in visual attention. Curr. Opin. Neurobiol. 7, 255-261.

Duncan, J., and Humphreys, G. W. (1989). Visual search and stimulus similarity. Psychol. Rev. 96, 433-458.

Fagot, C., and Pashler, H. (1995). Repetition blindness: perception or memory failure? J. Exp. Psychol. Hum. Percept. Perform. 21, 275-292.

Freeman, E., Sagi, D., and Driver, J. (2001). Lateral interactions between targets and flankers in low-level vision depend on attention to the flankers. Nat. Neurosci. 4, 1032-1036.

Han, S., Ding, Y., and Song, Y. (2002). Neural mechanisms of perceptual grouping in humans as revealed by high density event related potentials. Neurosci. Lett. 319, 29-32.

Han, S., Jiang, Y., Mao, L., Humphreys, G. W., and Gu, H. (2005a). Attentional modulation of perceptual grouping in human visual cortex: functional MRI studies. Hum. Brain Mapp. 25, 424-432.

Han, S., Jiang, Y., Mao, L., Humphreys, G. W., and Qin, J. (2005b). Attentional modulation of perceptual grouping in human visual cortex: ERP studies. Hum. Brain Mapp. 26, 199-209.

Humphreys, G. W. (1998). Neural representation of objects in space: a dual coding account. Philos. Trans. R. Soc. Lond., B, Biol. Sci. 353, 1341-1351.
Johnston, J. C., Hochhaus, L., and Ruthruff, E. (2002). Repetition blindness has a perceptual locus: Evidence from online processing of targets in RSVP streams. J. Exp. Psychol. Hum. Percept. Perform. 28, 477-489.

Kanwisher, N., Driver, J., and Machado, L. (1995). Spatial repetition blindness is modulated by selective attention to color or shape. Cogn. Psychol. 29, 303-337.

Kanwisher, N., Kim, J. W., and Wickens, T. D. (1996). Signal detection analyses of repetition blindness. J. Exp. Psychol. Hum. Percept. Perform. 22, 1249-1260.

Kanwisher, N. G. (1987). Repetition blindness - Type recognition without token individuation. Cognition 27, 117-143.

Khoe, W., Freeman, E., Woldorff, M. G., and Mangun, G. R. (2006). Interactions between attention and perceptual grouping in human visual cortex. Brain Res. 1078, 101-111.

Koffka, K. (1999). Principles of Gestalt Psychology. New York, Routledge.

Koivisto, M., and Revonsuo, A. (2008). Comparison of event-related potentials in attentional blink and repetition blindness. Brain Res. 1189, 115-126.

Lamy, D., Segal, H., and Ruderman, L. (2006). Grouping does not require attention. Percept. Psychophys. 68, 17-31.

Loftus, G. R., and Masson, M. E. (1994). Using confidence intervals in withinsubject designs. Psychon. Bull. Rev. 1, 476-490.

Mack, A., Tang, B., Tuma, R., Kahn, S., and Rock, I. (1992). Perceptual organization and attention. Cogn. Psychol. 24, 475-501.

Mattingley, J. B., Davis, G., and Driver, J. (1997). Preattentive filling-in of visual surfaces in parietal extinction. Science 275, 671-674.
Moore, C. M., and Egeth, H. (1997) Perception without attention: evidence of grouping under conditions of inattention. J. Exp. Psychol. Hum. Percept. Perform. 23, 339-352.

Morris, A. L., and Harris, C. L. (2004). Repetition blindness: out of sight or out of mind? J. Exp. Psychol. Hum. Percept. Perform. 30, 913-922.

Nikolaev, A. R., Gepshtein, S., Kubovy, M., and van Leeuwen, C. (2008). Dissociation of early evoked cortical activity in perceptual grouping. Exp. Brain Res. 186, 107-122.

Park, J., and Kanwisher, N. (1994). Determinants of repetition blindness. J. Exp. Psychol. Hum. Percept. Perform. 20, 500-519.

Pashler, H. (1987). Target-distractor discriminability in visual search. Percept. Psychophys. 41, 285-292.

Ptak, R., and Schnider, A. (2005). Visual extinction of similar and dissimilar stimull evidence for level-dependent attentional competition. Cogn. Neuropsychol. 22, 111-127.

Rafal, R., Danziger, S., Grossi, G., Machado, L., and Ward, R. (2002). Visual detection is gated by attending for action: evidence from hemispatial neglect. Proc. Nat. Acad. Sci. 99, 16371-16375.

Rafal, R., Ward, R., and Danziger, S. (2006). Selection for action and selection for awareness: evidence from hemispatial neglect. Brain Res. 1080, 2-8.

Roelfsema, P. R. (2006). Cortical algorithms for perceptual grouping. Annu. Rev. Neurosci. 29, 203-227.

Schendan, H. E., Kanwisher, N. G., and Kutas, M. (1997). Early brain potentials link repetition blindness, priming and novelty detection. Neuroreport 8 , 1943-1948.

Treisman,A. (1982). Perceptual grouping and attention in visual search for features and for objects. J. Exp. Psychol. Hum. Percept. Perform. 8, 194-214. van der Heijden, A. H. C., Malhas, M. S M., and van den Roovaart, B. P. (1984). An empirical interletter confusion matrix for continuous-line capitals. Percept. Psychophys. 35, 85-88.

Vuilleumier, P. O., and Rafal, R. D. (2000). A systematic study of visual extinction - Between- and withinfield deficits of attention in hemispatial neglect. Brain 123, 1263-1279.

Ward, R., Goodrich, S. J., and Driver, J. (1994). Grouping reduces visual extinction: Neuropsychological evidence for weight-linkage in visual selection. Vis. Cogn. 1, 101-129.

Wertheimer, M. (1923). Untersuchungen zur Lehre von der Gestalt: II. Psychol. Res.-Psychol. Forsh. 4, 301-350.

Wu, Y. H., Chen, J., and Han, S. H. (2005). Neural mechanisms of attentional modulation of perceptual grouping by collinearity. Neuroreport 16, 567-570.

Conflict of Interest Statement: The authors declare that the research was conducted in the absence of any commercial or financial relationships that could be construed as a potential conflict of interest.

Received: 14 July 2009; paper pending published: 02 October 2009; accepted: 15 February 2010; published online: 08 March 2010.

Citation: de Haan B and Rorden C (2010) Similarity grouping and repetition blindness are both influenced by attention. Front. Hum. Neurosci. 4:20. doi: 10.3389/fnhum.2010.00020

Copyright (C) 2010 de Haan and Rorden. This is an open-access article subject to an exclusive license agreement between the authors and the Frontiers Research Foundation, which permits unrestricted use, distribution, and reproduction in any medium, provided the original authors and source are credited. 